\title{
Correlating Radiation Exposure with Embrittlement: Comparative Studies of Electron- and Neutron-Irradiated Pressure Vessel Alloys*
}

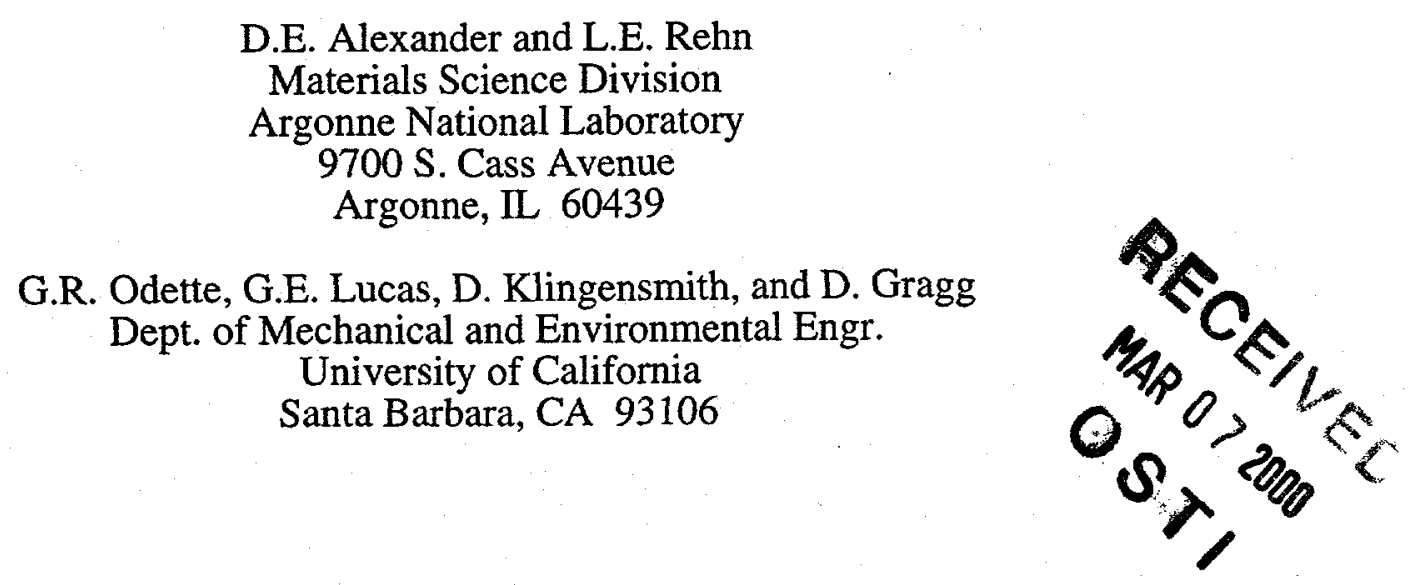

To be submitted to the $10^{\text {th }}$ International Symposium on Reactor Dosimetry, Osaka, Japan, September 12-17, 1999.

\footnotetext{
The submitted manuscript has been created by the University of Chicago as Operator of Argonne National Laboratory ("Argonne") under Contract No. W-31-109-ENG-38 with the U.S. Department of Energy. The U.S. Government retains for itself, and others acting on its behalf, a paid-up, nonexclusive, irrevacable worldwide license in said article to reproduce, prepare derivative works, distribute copies to the public, and perform publicly and display publicly, by or on behalf of the Government.
}

This work was supported by the U.S. Department of Energy, under contracts BES-MS and CTRMICS W-31-109-ENG-38. 


\section{DISCLAIMER}

This report was prepared as an account of work sponsored by an agency of the United States Government. Neither the United States Government nor any agency thereof, nor any of their employees, make any warranty, express or implied, or assumes any legal liability or responsibility for the accuracy, completeness, or usefulness of any information, apparatus, product, or process disclosed, or represents that its use would not infringe privately owned rights. Reference herein to any specific commercial product, process, or service by trade name, trademark, manufacturer, or otherwise does not necessarily constitute or imply its endorsement, recommendation, or favoring by the United States Government or any agency thereof. The views and opinions of authors expressed herein do not necessarily state or reflect those of the United States Government or any agency thereof. 


\section{DISCLAIMER}

Portions of this document may be illegible in electronic image products. Images are produced from the best available original document. 
Alexander, D. E. ', Kestel, B. J. ', Rehn, L. E. , Odette, G.R. ${ }^{2}$, Lucas, G.E' ${ }^{2}$, Klingensmith, D. ${ }^{2}$, Gragg, D. ${ }^{2}$, Seifert, S. ${ }^{3}$, and Jemian, P.R. ${ }^{4}$

\section{Correlating Radiation Exposure with Embrittlement: Comparative Studies of Electron- and Neutron-Irradiated Pressure Vessel Alloys}

Reference: Alexander, D. E. ${ }^{1}$, Kestel, B. J. ${ }^{1}$, Rehn, L. E. ${ }^{1}$, Odette, G.R. ${ }^{2}$, Lucas, G.E. ${ }^{2}$, Klingensmith, D. ${ }^{2}$, Gragg, D. ${ }^{2}$, Seifert, S. ${ }^{3}$, and Jemian, P.R. ${ }^{4}$, "Correlating Radiation Exposure with Embrittlement: Comparative Studies of Electron- and NeutronIrradiated Pressure Vessel Alloys," Reactor Dosimetry, ASTM STP \#\#\#, John G. Williams, David W. Vehar, Frank H. Ruddy and David M. Gilliam, Eds., American Society for Testing and Materials, West Conshohoken, PA, 2000.

Abstract: Comparative experiments using high energy (10 MeV) electrons and test reactor neutrons have been undertaken to understand the role that primary damage state has on hardening (embrittlement) induced by irradiation at $300^{\circ} \mathrm{C}$. Electrons produce displacement damage primarily by low energy atomic recoils, while fast neutrons produce displacements from considerably higher energy recoils. Comparison of changes resulting from neutron irradiation, in which nascent point defect clusters can form in dense cascades, with electron irradiation, where cascade formation is minimized, can provide insight into the role that the in-cascade point defect clusters have on the mechanisms of embrittlement. Tensile property changes induced by $10 \mathrm{MeV}$ electrons or test reactor neutron irradiations of unalloy ed iron and an $\mathrm{Fe}-0.9 \mathrm{wt} . \% \mathrm{Cu}-1.0 \mathrm{wt} . \% \mathrm{Mn}$ alloy were examined in the damage range of $9.0 \times 10^{-5}$ dpa to $1.5 \times 10^{-2} \mathrm{dpa}$. The results show the ternary alloy experienced substantially greater embrittlement in both the electron and neutron irradiated samples relative to unalloy ed iron. Despite their disparate nature of defect production, similar embrittlement trends with increasing radiation damage were observed for electrons and neutrons in both the temary and unalloyed iron.

Keywords: damage correlation, neutron irradiation, electron irradiation, pressure vessel embrittlement, primary damage state, small-angle $\mathrm{x}$-ray scattering

\footnotetext{
${ }^{1}$ Materials Science Division, Argonne National Laboratory, 9700 S. Cass Ave., Argonne, IL, 60439.

${ }^{2}$ Department of Mechanical and Environmental Engineering, University of CaliforniaSanta Barbara, Santa Barbara, CA, 93106.

${ }^{3}$ Chemistry Division, Argonne National Laboratory.

${ }^{4}$ Materials Research Laboratory, University of Illinois at Urbana-Champaign, UNICAT, Argonne, IL 60439.
} 


\section{INTRODUCTION}

Radiation-induced embrittlement of nuclear reactor pressure vessels (RPVs) is a complex process involving a variety of environmental variables (irradiation flux, fluence, temperature, etc.) and metallurgical variables (alloy composition, pre-existing microstructure, etc.) [1]. Rate theory ap proaches have been used to incorporate these various variables and model the evolution of microstructural features such as alloy phases [e.g. copper-rich precip itates (CRPs)] and defect clusters [1-4] with fluence. Embrittlement is caused by yield stress increases introduced by these hardening centers (hence the terms "embrittlement" and "hardening" are used interchangeably in the remainder of the paper). As available computing power increases, atomistic simulations of microstructural evolution such as molecular dy namics (MD) and kinetic Monte Carlo have become more practical [5-10]. While offering the potential to eventually supplant mean-field rate theory, these approaches are in their early application stages and are subject to current computational and phy sical limitations (e.g inadequate interatomic potentials, limited simulation cell size and simulation time, incomplete rep roduction of measured phy sical properties, etc.).

With the goal of better modeling radiation effects of interest to embrittlement a hybrid ap proach has evolved in which the nascent defect production results of MD simulations have been used as input to rate theory models $[1-4,11]$. MD simulations in pure $\mathrm{Fe}$, over the time scale of many of picoseconds, show that primary defect production varies with the energy of the initiatingrecoil atom. High-energy, neutron-induced recoil cascades induce the formation of point defect clusters (ak.a. cascade remnants) and a reduction in the total number of defects relative to NRT predictions. In contrast, low-energy recoils result in less clustering and increased total defect survival approaching that predicted by NRT.

Exp erimentally this "spectral effect" (i.e. the effect of variations in primary defect production caused by variations in atomic recoil energy ) has been well-documented in experiments involving defect-mediated mass transport phenomena such as radiation-induced segregation [12], radiation-enhanced diffusion [13] and void swelling [14]. However, the impact of nascent defect production variations on embrittlement is not immediately clear. Potential effects were explored in rate-theory modeling by Stoller [4] who showed that modest variations in primary defect production parameters such as interstitial/vacancy cluster fractions and defect production efficiency could affect the magnitude of predicted embrittlement substantially.

As described previously [15], another motivation for understanding the role of differences in defect production stems from the interest in gamma ray embrittlement. Gamma ray s leaking from the core of the reactor interact with the RPV producing defects indirectly via photoelectron processes. In certain reactors, the contribution of gamma ray $\mathrm{s}$ to total damage experienced by the RPV can be significant when calculated on a dpa basis. The high energy electrons (or positrons), produced by the gamma rays produce displacement through low energy atomic recoil transfers. As such their contribution to microstructural changes inducing embrittlement may be different than that generated by the high energy recoil cascades of fast neutrons. 
Given the sensitivity of the modeling to input derived from MD simulations and the potential contribution of gamma-rays, experimental evaluation of the impact of the primary damage state on embrittlement is desirable. With this in mind, experiments have been undertaken to compare fast neutron and electron irradiation effects on embrittlement. Electrons produce disp lacement damage primarily by low energy atomic recoils, while fast neutrons produce displacements from considerably higher energy recoils. Defect production differences between the two ty pes of irradiat ing particles can be quantified by examining their primary recoil damage spectra [16]. The median recoil damage energy, $T_{1 / 2}$, the energy above and below which half the displacement damage is produced, is nearly two orders of magnitude smaller for $\mathrm{MeV}$ electron irradiation than for fast neutron irradiation. Comparison of changes resulting from neutron irradiation, in which nascent point defect clusters can form in dense cascades, with electron irradiation, where cascade formation is minimized, can provide insight into the role that the in-cascade point defect clusters have on the mechanisms of embrittlement.

\section{EXPERIMENTS}

Miniature sheet tensile specimens with a gage section area of $9 \mathrm{~mm} \times 2 \mathrm{~mm}$ were prepared by precision die punching heat-treated wafers that had been lapped to $0.5 \mathrm{~mm}$ final thickness. The annealing treatment for both the Fe-only and $\mathrm{Fe}-0.9 \mathrm{wt} . \% \mathrm{Cu}-1.0 \mathrm{wt} . \% \mathrm{Mn}$ model alloy prior to wafer thinning and punching consisted of annealing at $775^{\circ} \mathrm{C}$ for 17 hours followed by a salt quench at $450^{\circ} \mathrm{C}$ for 3 minutes (no subsequent temper or stress relief). This treatment resulted in an average grain diameter of around $75 \mu \mathrm{m}$ in the ternary alloy and one slightly larger in unalloy ed $\mathrm{Fe}$.

Both model alloy and Fe tensile samp les were electron irradiated with $10-\mathrm{MeV}$ electrons from a pulsed linear accelerator op erating at $60 \mathrm{~Hz}$. The slightly defocused beam entered a helium backfilled target chamber held at 0.7 atm and passed through a $12 \mathrm{~mm}$ square, watercooled collimator which defined the irradiation area on the gage section of the samples. To assist irradiation uniformity over the gage sections, the electron beam was wobbled slightly about $\pm 2 \mathrm{~mm}$ at rates of $4.5 \mathrm{~Hz}$ and $0.7 \mathrm{~Hz}$ in the horizontal and vertical directions, respectively. Four samples stacked two deep allowed for 8 tensile specimens to be irradiated in a single bading. Samples were heated by the electron beam and the current required to maintain the center gage section at the target temperature of $300^{\circ} \mathrm{C}$ was about 18 $\mu \mathrm{A}$. For a medium $\mathrm{Z}$ material, assuming a damage cross section of 96 barns (based on Oen's compilations [17] assuming a threshold disp lacement energy of $40 \mathrm{eV}$ and with correction for energy loss), this corresponded to a damage rate of $7.5 \times 10^{-9} \mathrm{dpa} / \mathrm{s}$. In order to monitor temperature, one of the specimens was outfitted with ty $\mathrm{pe}-\mathrm{K}$ thermocouples spot-welded at the center of, and just outside the top and bottom extent of the gage section. During the irradiation, a temperature gradient was present across the length of the samples with the ends of the gage section ty pically about $25^{\circ} \mathrm{C}$ cooler than the center held at $300^{\circ} \mathrm{C}$.

The same type of mini-tensile specimens were irradiated with fast neutrons at $290^{\circ} \mathrm{C}$ in two different experiments in the Ford Nuclear Reactor at the University of Michigan. The more recent experiment used the Irradiation Variable Faclity (IVAR) which was constructed 
in collaboration with University of California-Santa Barbara, Oak Ridge National Laboratory and the US Nuclear Regulatory Commission. This experiment involves an extensive test matrix including a variety of model alloys subjected to three different damage rates and irradiation temperatures. A higher fluence set of data from an older, "piggy back" experiment is also included here. In both experiments, samples were in aluminum cap sules in a dry inert gas environment during the irradiation and held in the pool region immediately adjacent to the east face of the reactor core. The IVAR facility allows continuous monitoring of temperature and power during reactor cy cles lasting 10 days. While Fe and Ni dosimeter wires were included in each capsule, they have not y et been analy zed and the flux and fluence values used in this work are based on a prior, preliminary dosimetric evaluation of the IVAR region. Neutron damage rates for this experiment are listed in Table 1 and were determined assuming a spectral- averaged damage cross section of 1500 barns.

Samples were tensile tested at room temperature at a strain rate of $10^{-3} \mathrm{~s}^{-1}$. At least two specimens of each fluence and alloy were tested, with the exception of the electron irradiated Fe-only samples where only one specimen of each fluence was used. Unirradiated, control specimens, associated with the same alloy forging locations as their corresponding irradiated counterparts, were also tested.

Table 1. Experimental Damage Rates

\begin{tabular}{|c|c|c|}
\hline & $\begin{array}{c}\text { Damage Rate } \\
(\mathrm{dpa} / \mathrm{s})\end{array}$ & $\begin{array}{c}\text { Ratio relative to IVAR } \\
\text { Low Neutron }\end{array}$ \\
\hline IVAR Low Neutron & $9.0 \times 10^{-11}$ & 1 \\
\hline IVAR Medium Neutron & $4.7 \times 10^{-10}$ & 5 \\
\hline Piggy-Back Neutron & $1.0 \times 10^{-9}$ & 11 \\
\hline IVAR High Neutron & $1.4 \times 10^{-9}$ & 83 \\
\hline Linac Electrons & $7.5 \times 10^{-9}$ & \\
\hline
\end{tabular}

Small-angle $\mathrm{x}$-ray scattering experiments (SAXS) have been undertaken to characterize the irradiated alloys . SAXS provides a useful complement to microscopic techniques including electron microscopy and atom probe for characterizing irradiated microstructures. By sampling a large number of hardening centers in the material they can provide statistically quantitative size and number density information on the very fine scale embrittled microstructures that form subsequent to low damage level irradiation of ferritic alloys of interest to reactor pressure vessels. Details of the experimental technique may be found in ref. [18]. Since the publication of this previous work, substantial improvements have been made which improve the collection efficiency and quality of SAXS data A charge-ooup led device camera is now used to collect SAXS intensity best leveraging 
the intense photons fluxes available at the Advanced Photon Source. The use of a vacuum sample target chamber greatly improved signal to background noise detection enhancing the ability to detect weak SAXS signals.

\section{RES ULTS AND DISCLSSION}

\section{Mechanical Property Testing}

Fig. 1 shows the change in yield strength (YS) as a function of calculated dp a for both the electron- and neutron-irradiated samples. Display ed tensile property eror bars were derived from the standard deviations of the respective irradiated and unirradiated measurements. Error bars of $\pm 20 \%$ were attached to the neutron data damage level data to approximately account for uncertainties in dosimetry estimates.

The first observation readily apparent from Fig. 1 is, for the same damage level, the ternary alloy experienced substantially more embrittlement than uanlloyed $\mathrm{Fe}$ regardless of the irradiating species. The pronounced effect on embrittlement of alloying species has been well-documented in previous neutron irradiation experiments [1]. Similar behavior has also been reported in recent work examining electron irradiation-induced hardening.

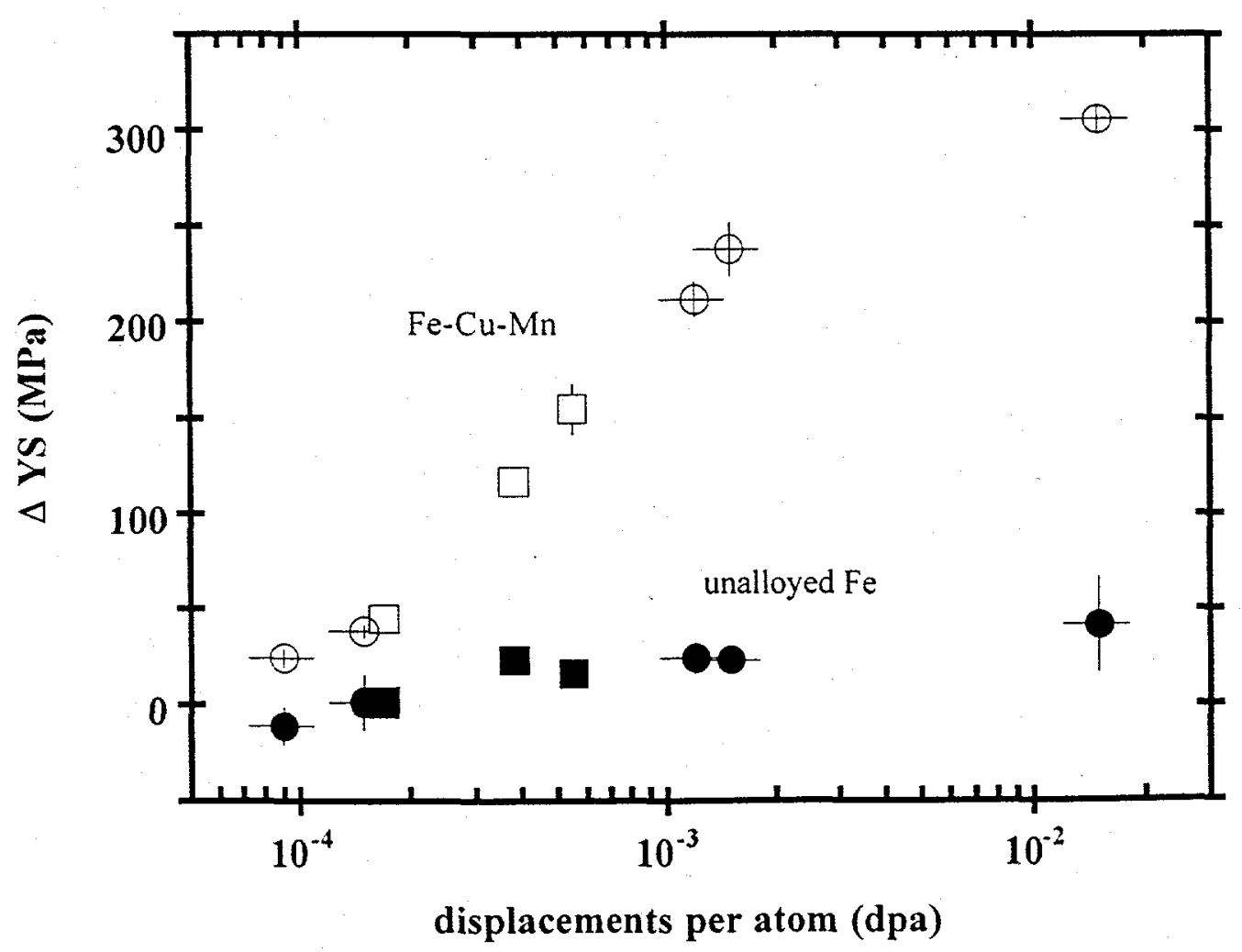

Fig. I Change in yield strength (YS) measured at room temperature as a function of calculated irradiation damage (dpa) at $300^{\circ} \mathrm{C}$. Squares are the $10-\mathrm{MeV}$ electron data, and circles are fast neutron test reactor data. 
Barbu et al. [19] saw no increase in microhardness in pure Fe irradiated at $290^{\circ} \mathrm{C}$ with 2.5 $\mathrm{MeV}$ electrons to about $4.2 \times 10^{-4}$ (at a rate of $2 \times 10^{-9} \mathrm{dpa} / \mathrm{s}$ ) while an $\mathrm{Fe}-0.3$ at.\% $\mathrm{Cu}$ alloy subjected to the same irradiation conditions experienced a $60 \%$ increase in hardness.

Microstructurally, the increased embrittlement in the alloy is typically attributed to the diffusive nucleation and growth of pure $\mathrm{Cu}$ or $\mathrm{CRPs}$ which provide an additional hardening component. Small-angle neutron scattering (SANS) experiments [1] and recent electron microscopy work are consistent with this CRP interpretation. High resolution electron microscopy (HREM) in higher $\mathrm{Cu}$-content $(\geq 1.3 \mathrm{wt} . \% \mathrm{Cu})$ binary alloys shows evidence for pure $\mathrm{Cu}$ precipitates forming subsequent to electron- or neutron-irradiation $[20,21]$. Reactor neutron irradiation at $270^{\circ} \mathrm{C}$ to damage levels of $8.61 \times 10^{-3} \mathrm{dpa}$ and $6.3 \times 10^{-2}$ dpa resulted in mostly spherical precipitates with similar diameter ranges of 2-4 $\mathrm{nm}$. HREM work on binary alloy irradiated at $295^{\circ} \mathrm{C}$ with $2.5 \mathrm{MeV}$ electrons to $1.4 \times 10^{-3}$ $\mathrm{dpa}$ (at a rate of $2 \times 10^{-9} \mathrm{dpa} / \mathrm{s}$ ) also show pure $\mathrm{Cu}$ precipitates with the same $9 \mathrm{R}$ twinned structure observed in neutron irradiated and in the annealed-only alloy. The microstructural similarity with thermally annealed material supports the idea that irradiation, whether by electrons or neutrons, enhances the precipitation process by increasing the point defect concentrations mediating thermal diffusion. While HREM is not sensitive to the coherent $\mathrm{BCC}$ structure believed characteristic of small $\mathrm{Cu}$ precipitate sizes in this binary alloy, the larger diameter range of the non-coherent precipitates observed at lower damage level in the electron irradiated material $(4-17 \mathrm{~nm})$ versus that for the higher damage level neutron irradiated alloy $(2-4 \mathrm{~nm})$ is consistent with the notion that electrons are more efficient than neutrons at promoting radiation-enhanced diffusion.

A second observation from Fig. 1 is the similarity in tensile property change trends with radiation damage for electrons and neutrons. This same embrittling efficiency per dpa for both irradiating species remains qualified by the fact that different damage rates were used in the various experiments. As seen in Table 1, there exists a factor of 83 difference between the highest damage rate (linac electrons) and the lowest neutron damage rate. Among only the neutron irradiations, a more modest factor of 16 difference exists between the highest and lowest damage rates.

A more sound experimental basis upon which to comment on damage rate effects will be available in the near future with additional IVAR neutron test matrix data. In the interim however, damage rate effect differences are discounted by considering model predictions and additional existing data from the neutron irradiation test matrix. Although rate theory modeling has not been applied to analyze the specific results of our experiment, the cluster-recombination rate theory model of Odette [1] suggests that there should be only a minimal to modest effect on hardening for this difference, at the damage rate levels studied.

Assessing damage rate effects on embrittlement trends for electrons is less straight forward in lieu of experimental data or comprehensive modeling. A simple, qualitative approach would be to assume that during electron irradiation embrittlement evolves solely from diffusion of point defects which contribute to formation of defect clusters and CRPs. Steady-state rate theory would then predict that at low damage rates, fixed-sink (e.g. dislocation) dominated kinetics would ensue and the embrittlement rate (mechanical 
property change per dpa) would be constant, independent of the electron damage rate (similar to the neutron behavior described above). In the limit of high damage rates, mutual recombination of point defects dominates the kinetics and the embrittlement rate would decrease with increasing damage rate according to (damage rate) ${ }^{-1 / 2}$.

With these interpretations of damage rate effects, we can identify two possible qualitative conclusions regarding the embrittling efficiencies of the electrons and neutron irradiations used in this work. If the electron irradiation damage rate is considered to be in the damage rate independent regime, the experimental data simply implies that electrons and neutrons are equally efficient at producing embrittlement even when extrapolated down to the low damage rate levels of interest to commercial light water RPVs. If however, we consider the electron damage rate to be in the damage rate dependent regime, it becomes necessary to "correct" for embrittlement rate changes due to damage rate prior to comparing the two types of irradiating species. Taking into account an anticipated embrittlement rate decrease due to high damage rate, the fact that the electron-induced tensile changes follow the same trend as those induced by the lower damage rate neutrons (Fig. 1) suggests that the electrons are more efficient (on a per dpa basis) than the neutrons at causing embrittlement when extrapolated down to the low damage rates of interest to commercial light water RPVs.

The results for this experiment indicate that the substantial primary damage state differences known to exist between electrons and neutrons do not always exert a corresponding influence on the aggregate changes in mechanical properties. This may suggest that future modeling efforts must carefully consider the balance of competing effects that may be produced by various types of primary sources of displacement damage on all the multiscale-multiphysics processes [22] leading to hardening and embrittlement.

\section{Microstructire Characterization}

Clearly, further understanding the similarity in embrittlement behavior between electrons and neutrons awaits detailed microstructural characterization of the irradiation embrittled materials. In this regard, experiments have been undertaken to use SAXS to characterize irradiated microstructures. Fig. 2 shows the contrast-weighted number density distributions determined from a maximum entropy analysis of SAXS data collected at three energies near the $\mathrm{Fe}-\mathrm{K}, \mathrm{x}$-ray absorption edge. Despite the very low damage level, $5 \times 10^{-4} \mathrm{dpa}$, small (11.8 $\AA$ average radius) hardening centers are clearly observed in this electron irradiated material. The increasing distribution height with photon energy is a result of an anomalous scattering effect in which the contrast between the scattering centers and the surrounding matrix increases as the photon energy increases. In the future, by examining the energy dependence of the integrated distribution area, at this edge as well as those of the other constituent elements in the alloy, hardening center chemical composition will be derived. 


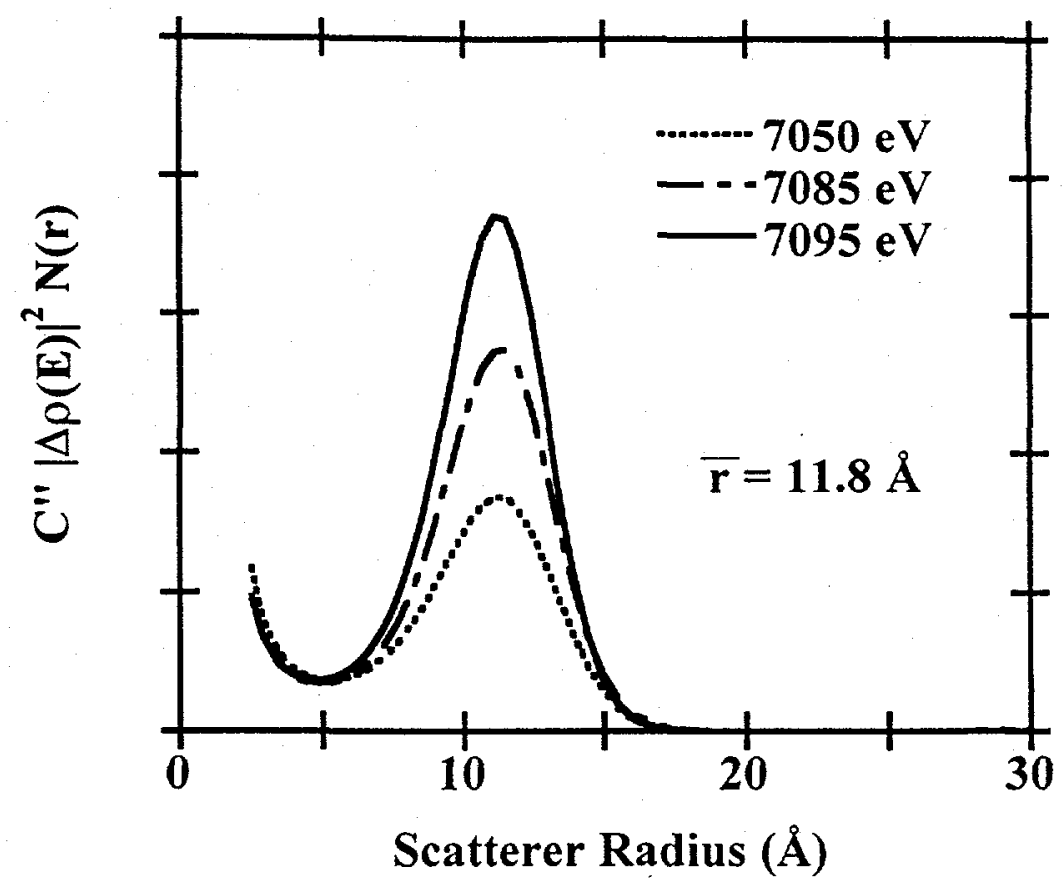

Fig. 2 Contrast-weighted, hardening center size distributions for the $\mathrm{Fe}-\mathrm{Cu}$-Mn alloy electron irradiated to $5 \times 10^{-4}$ dpa determined through maximum entropy analysis of $S A X S$ data collected at three photon energies near the $\mathrm{Fe}-\mathrm{K}$ absorption edge.

\section{CONCLUSIONS}

Changes in y ield strength and ultimate tensile strength resulting from $10 \mathrm{MeV}$ electronor test reactor neutron-irradiation at $300^{\circ} \mathrm{C}$ revealed a strong effect of small alloying additions to iron Regardless of the irradiating species, an $\mathrm{Fe}-0.9 \mathrm{wt} . \% \mathrm{Cu}-1.0 \mathrm{wt} . \% \mathrm{Mn}$ alloy experienced substantially greater tensile changes compared to unalloy ed $\mathrm{Fe}$ in the damage range of $9.0 \times 10^{-5} \mathrm{dpa}$ to $1.5 \times 10-2 \mathrm{dpa}$. This result is consistent with previous work demonstrating the addition of a Cu-related precipitation component of hardening in the alloy. The observation of similar embrittlement trends with dpa for the electron and neutron irradiation, when interpreted with respect to the different damage rates emp loy ed in the experiments, led to the conclusion that the efficiency for electrons to cause embrittlement was greater than, or equal to that of the neutrons when extrapolated to the low damage rate levels characteristic of commercial light water RPVs.

\section{ACKOWLEDGMENTS}

This work was supported in part by the U.S. Department of Energy, BES-Materials Sciences, under contract \#W-31-109-ENG-38. The use and support provided by the BESSRC CAT at the Advanced Photon Source is gratefully acknowledged. 


\section{REFERENCES}

1. G.R. Odette, and G.E. Lucas, Radiation Effects and Defects in Solids, 144 (1998), 189231.

2. G.R. Odette, in Microstructure of Irradiated Materials, Vol. 373, ed I. M. Robertson, L. E. Rehn, S. J.Zinkle and W. J. Phy thian (Materials Research Society, Pittsburgh, PA, 1995), 137-148.

3. E.V.Mader., "Kinetics of Irradiation Embrittlement and the Post-Irradiation Annealing of Nuclear Reactor Pressure Vessel Steels" (Ph.D. Thesis, University of California, Santa Barabara, 1995).

4. R.E. Stoller, "A Comparison of the Relative Importance of Copper Precipitates and Point Defect Clusters in Reactor Pressure Vessel Embrittlement", Oak Ridge National Laboratory NUREGKR-6231, 1994.

5. R. E Stoller,, G.R. Odette and B.D. Wirth, Joumal of Nuclear Materials 251 (1997), 4960.

6. W.J. Phy thian, R.E. Stoller, A J.E. Foreman, A.F. Calder and D.J. Bacon, Journal of Nuclear Materials 223 (1995), 245-261.

7. N. Soneda and T. Dizz de la Rubia, Philosophical Magazine A 78 (1998), 995-1019.

8. F. Gao, D.J. Bacon, P.E.J. Flewitt and T.A. Lewis, in Microstructure Evolution During Irradiation, Vol. 439, ed I.A. Robertson, G.S. Was, L.W. Hobbs and T.D. de la Rubia (Materials Research Society, Pittsburgh, PA, 1997), 307-318.

9. A.F. Calder and D.J. Bacon, ibid, 521-526.

10. D.J. Bacon, A.F. Calder and F. Gao, Journal of Nuclear Materials 251 (1997), 1-12.

11. Y. Katoh and T. Muroga, Journal of Nuclear Materials 233-237 (1996), 1029-1034.

12. L.E. Rehn and H. Wiedersich, Materials Science Forum 97-99 (1992), 43-58.

13. H. Wolknberger, V.Naundorf, and M.-P. Macht, "Radiation-Induced Diffusion in Nuclear Materials" in Diffusion Processes in Nuclear Materials, ed R.P. Agarwala (Elsevier Science Publishers B.V., Amsterdam, The Netherlands, 1992).

14. L.K. Mansur and P.R. Okamoto, Nuclear Metallurgy 18 (1973), 509.

15. D. E Alexander and L.E. Rehn, "The Role of Gamma Rays and Frely-Migating Defects in Reactor Pressure Vessel Embrittlement", in Proceedings of the 9th International Sy mposium on Reactor Dosimetry, edited by H.A. Abderrahim, P. D'Hondt and B. Osmera (World Scientific Publishing, Singapore, 1998), pp. 508-515.

16. R.S. A verback, Journal of Nuclear Materiak 216 (1994), 49-62.

17. O.S. Oen, "Cross Sections for Atomic Displacements in Solids by Fast Electrons", Oak Ridge National Laboratory ORNL-4897, 1973.

18. D. E. Alexander, B.J. Kestel, P.R. Jemian, G.R Odette, D. Klingensmith and D. Grag, "Anomalous Small-Angle X-Ray Scattering Characterization of Model Pressure Vessel Alloy Microstructures", in Microstructural Processes in Irradiated Materials, Vol. 540, edited by S.J. Zinkle, G.E. Lucas, R.C. Ewing and J.S. Williams (Materials Research Society, Warrendale, PA, 1999), pp. 415-463.

19. A. Barbu, M.H. Mathon, F. Maury, J.F. Belliard, B. Beuneu and C.H. de Novion, Joumal of Nuclear Materials 257 (1998), 206-211. 
20. H.A. Hardouin Duparc, R.C. Dook, M.L. Jenkins and A. Barbu, Philosophical Magazine Laters 71 (1995), 325-333.

21. A.C. Nicol, M.L. Jenkins and M.A. Kirk, in Microstructural Processes in Irradiated Materials, Vol. 540, ed S.J. Zinkle, G.E. Lucas, R.C. Ewing and J.S. Williams (Materials Research Society, to be published 1999), 409-414.

22. These processes, which range from primary defect production, through nanostructural evolution to structure property relations, are discussed in Reference [1]. 